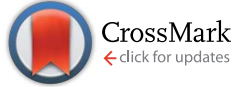

Cite this: J. Anal. At. Spectrom., 2016, 31,1647

\title{
Linearity testing and dead-time determination for MC-ICP-MS ion counters using the IRMM-072 series of uranium isotope reference materials
}

\author{
S. Richter, ${ }^{\text {a }}$ S. Konegger-Kappel, ${ }^{\text {b }}$ S. F. Boulyga, ${ }^{b}$ G. Stadelmann, ${ }^{\text {b } A . ~ K o e p f ~}{ }^{\text {b }}$ \\ and $\mathrm{H}$. Siegmund ${ }^{\mathrm{b}}$
}

The Environmental Sample Laboratory (ESL) of the International Atomic Energy Agency (IAEA) routinely analyses environmental swipe samples for their bulk $U$ and $\mathrm{Pu}$ isotope amounts and ratios using a Neptune Plus ${ }^{\mathrm{TM}}$ (Thermo Fisher Scientific) multi collector-inductively coupled plasma mass spectrometer (MC-ICP-MS). The instrument is equipped with the so-called "L5 ion counter package", which comprises ten Faraday cups, three classical discrete dynode secondary electron multipliers (SEM), and two compact discrete dynode (CDD) electron multipliers. In contrast to classical SEMs, CDDs only have about twice the width of a Faraday cup and are mounted in-line with the Faraday cups within the multi collector array. The Institute of Reference Materials (IRMM) of the European Commission's Joint Research Centre (JRC) has developed several dedicated uranium isotope reference material series for the investigation of non-linearity and dead time effects of ion counting systems. For instance, a new series of gravimetrically prepared uranium isotope reference materials, the so-called IRMM-074 series, with the ${ }^{235} \mathrm{U} /{ }^{238} \mathrm{U}$ isotope ratio held constant at unity and the ${ }^{233} \mathrm{U} /{ }^{235} \mathrm{U}$ isotope ratios varying from 1.0 to $10^{-6}$ has been prepared and certified. This series is suited for calibration of secondary electron multipliers used widely in isotope mass spectrometry, in particular for thermal ionization mass spectrometry (TIMS), ICP-MS and accelerator mass spectrometry (AMS). The new IRMM-074 series was prepared as a replacement for the already exhausted IRMM-072 predecessor series, which is still on stock at the IAEA. In collaboration between the IAEA and the IRMM, dedicated new procedures have been developed for taking advantage of the IRMM reference materials for linearity testing and dead time determination for the various ion counting detectors of two Neptune Plus ${ }^{T M}$ MC-ICP-MS instruments at the IAEA. Only statically measured ratios were used, and dynamic (peak-jumping) ratios were avoided, which makes these procedures independent on plasma instabilities and therefore ideal for MC-ICP-MS instruments. The dead times of the ion counting systems were found to depend not on the detectors themselves but only on the pulse amplifiers, which allowed to easier investigate them by connecting each pulse amplifier to any detector of the same type within the detector configuration. The new procedures might well be applicable for similarly designed MC-ICP-MS instruments which have already been installed at other laboratories working in the nuclear safeguards and environmental fields.

Received 2nd June 2016 Accepted 1st July 2016

DOI: $10.1039 / c 6 j a 00203 j$ www.rsc.org/jaas measurements of nuclear materials. There are only four main providers of nuclear reference materials world-wide. The National Institute of Standards and Technology (NIST) and, since the late 1970 s for the nuclear elements uranium and plutonium, the New Brunswick Laboratory (NBL, US-DOE), the Commission d'ETAblissement des Méthodes d'Analyse du Commissariat à l' énergie atomique (CEA/CETAMA) and the European Commission - Joint Research Center - Institute for Reference Materials and Measurements (IRMM). In the European Union, JRC-IRMM is the recognized provider for nuclear isotope reference materials to the nuclear industry and nuclear safeguards authorities.

In isotope ratio mass spectrometry there is a strong need for certified isotopic reference materials (CRM) for instrument 
calibration and quality control. In particular for nuclear safeguards, the main elements of interest are uranium, plutonium, thorium and americium. The measurement of uranium by mass-spectrometry is a special challenge because of the very wide dynamic range of the isotope abundances typically found in nature as well as in samples of the nuclear fuel cycle. During the recent years a programme has been carried out at IRMM to replace the series of certified reference materials (CRM), IRMM-072, which was prepared about 30 years ago., IRMM-072 was prepared gravimetrically from purified, highly enriched uranium isotopic material of ${ }^{233} \mathrm{U},{ }^{235} \mathrm{U}$ and ${ }^{238} \mathrm{U}$. The IRMM-072 series consisted of 15 individual CRMs; in each of these the isotopic ratio ${ }^{235} \mathrm{U} /{ }^{238} \mathrm{U}$ was held constant at a value close to unity and ${ }^{233} \mathrm{U} /{ }^{235} \mathrm{U}$ varied in 15 steps across the series from 1.0 down to $10^{-6}$. The $\mathrm{U}$ concentration in IRMM-072 was $1 \mathrm{mg} \mathrm{U}$ per $\mathrm{mL}$ of solution for each of the 15 samples of the entire set, which was considered as a concentration for easy use in mass spectrometry laboratories.

In 2002, part of the remaining material of IRMM-072 was diluted in order to make this material also available for use in environmental uranium mass spectrometry. The U concentration of the diluted series, the so-called IRMM-073 series, ${ }^{3}$ was adjusted to $1.5 \mu \mathrm{g} \mathrm{U}$ per $\mathrm{mL}$ solution. The entire set has a total activity just below $1000 \mathrm{~Bq}$ in order to facilitate application and shipment for laboratories working in environmental fields.

Because of the usefulness and general popularity of the IRMM-072 series, it became clear some years ago that a replacement would have to be made and certified. The original methods had to be rediscovered and where necessary adapted to present conditions. In particular the dynamic range of $1: 10^{-6}$ was retained but fewer members in the set were deemed to be needed. The preparation and certification of this new series, the certified isotopic reference material IRMM-074, is described in detail in. ${ }^{4}$ The three series IRMM-072, IRMM-073 and IRMM-074 are suitable for the investigation of detector systems installed in mass spectrometers, in particular for SEM detectors. The linearity response of a detector can be determined by measuring the varying ${ }^{233} \mathrm{U} /{ }^{235} \mathrm{U}$ ratio across the series, while the ${ }^{235} \mathrm{U} /{ }^{238} \mathrm{U}$ isotopic ratios that are close to unity allow an ideal internal correction of the mass fractionation of any type of mass spectrometer.

In 1987, Rosman et al. ${ }^{1}$ described the use of IRMM-072 for SEM linearity testing and presented how far from ideal the behaviour of SEMs can actually be. Significant deviations of up to $3 \%$ for the measured ${ }^{233} \mathrm{U} /{ }^{235} \mathrm{U}$ ratios from the certified values were observed. But for this investigation the SEM was operated still in analogue mode, which excludes the dead time of any pulse counting system to be responsible for the observed deviations. Thus the non-linearity was originating in the SEM detector itself. But since sufficiently fast pulse counting systems have become available, for high precision isotope ratio measurements SEMs are usually operated in the pulse counting mode (also called ion counting mode) rather than in analogue mode. The advantage of the pulse counting mode is the much lower background noise and the smaller uncertainty arising from that. But due to the fact that SEM detectors may generally exhibit their own non-linearity effects, even for SEMs operated in ion counting mode, non-linearity effects cannot only be directly attributed to the dead-time of the pulse amplifier or pulse counting electronics. The often made assumption, that linearity testing of SEMs is equivalent with dead time determination has to be re-considered very carefully in each case.

The linearity of an SEM operated in ion counting mode is dependent on the linearity of two components, firstly the SEM detector itself and secondly the pulse counting system. A possibly third component, the dead time of the SEM itself is usually shorter (less than $10 \mathrm{~ns}$ ) and therefore completely covered by the dead time of the pulse amplifier (20-70 ns), which is often fixed electronically. In order to investigate the combined system of the SEM itself and the pulse counting system, first the dead time of the pulse amplifier has to be determined independently from the SEM detector, e.g. using electronic equipment. With application of this estimated dead time value, a linearity test of the SEM detector itself can be performed by measurements of certified reference materials like IRMM-072/-073/-074. If this test confirms the linearity of the detector by close agreement of the measured with the certified values, a more reliable and SI traceable dead time value can then be determined by performing a linear regression calculation on the dead-time-un-corrected measured isotope ratios for the certified reference materials.

A new generation of SEMs was developed from a collaboration of Thermo Fisher Scientific with MasCom in 2006. This new SEM has been investigated for linearity at IRMM as well. ${ }^{4}$ On two TIMS instruments the new SEMs were found to be linear within $0.1 \%$, and the dead time of the combined system of SEM and pulse amplifier was found to agree with the nominal value of $20 \mathrm{~ns}$ for the dead time of the pulse amplifier. Two procedures were introduced for the dead time determination, the so-called static procedure recommended for MC-ICP-MS instruments and the so-called dynamic procedure recommended for TIMS instruments.

\section{Experimental}

\section{The Neptune Plus ${ }^{\mathrm{TM}}$ MC-ICP-MS instruments at the IAEA}

The detector configuration of the Neptune Plus ${ }^{\mathrm{TM}}$ MC-ICP-MS that is currently installed at the Environmental Sample Laboratory of the International Atomic Energy Agency (IAEA) - in the following referred to as Neptune-1 - is shown in Fig. 1. The

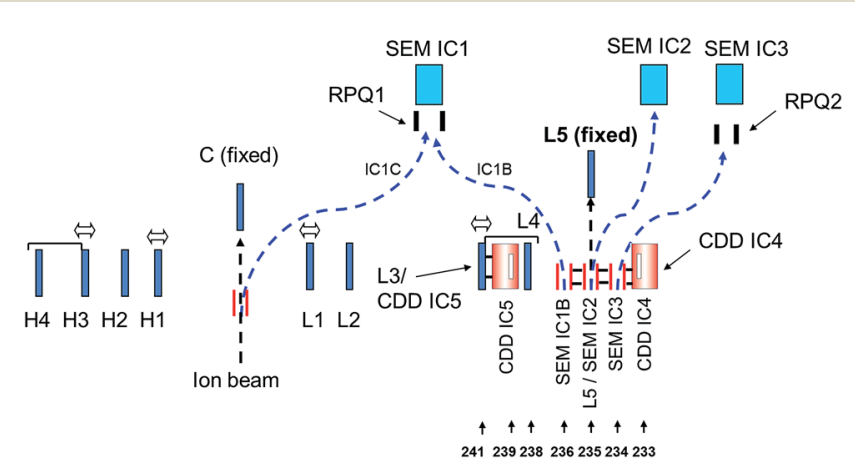

Fig. 1 Detector configuration of the Neptune Plus ${ }^{T M}-1$ MC-ICP-MS. 
conceptual design of the detector array for the Neptune Plus was developed and tested by Thermo Fisher in cooperation with the Pacific Northwest National Laboratory team in 2008-2009. ${ }^{5}$ The instrument is equipped with the so-called "L5 ion counter package", it includes ten Faraday cups, three classical standard sized discrete dynode secondary electron multipliers - in the following referred to as SEM detectors - and two compact discrete dynode electron multipliers (CDD). ${ }^{6}$ The CDD detectors with a width of 6-7 $\mathrm{mm}$ are mounted within the focal plane of the multi-collector along with the Faraday cups. They have a similar measurement performance as standard sized discrete dynode secondary electron multipliers, which have a larger width of about $20 \mathrm{~mm}$ and therefore have to be mounted in separate places within the detector housing. The CDD detectors allow simultaneous ion counting collection for isotopes in the $\mathrm{U}-\mathrm{Pu}$ mass region, with a mass difference of one amu on one but at least two amu on the other side of the detector. This is a disadvantage compared to the previously used "continuous dynode electron multipliers" (CDEM) which have about the same dimensions as the standard Faraday cups. On the other hand, the CDEM detectors have shown unsatisfactory performance in terms of stability, dynamic range and life time, compared to the CDD detectors. This was the reason for abandoning them for the use on Neptune Plus ${ }^{\mathrm{TM}}$ MC-ICP-MS and Triton Plus ${ }^{\mathrm{TM}}$ TIMS instruments.

Fig. 2 shows another Neptune Plus ${ }^{\mathrm{TM}}$ MC-ICP-MS which is installed at the Nuclear Material Laboratory (NML) of the International Atomic Energy Agency (IAEA) - in the following referred to as Neptune-2 - which has one more CDD detector included compared to Neptune-1, called CDD IC6.

The detector configurations of Neptune Plus ${ }^{\mathrm{TM}}-1$ MC-ICP-MS and Neptune Plus ${ }^{\mathrm{TM}}-2$ MC-ICP-MS instruments were designed on the basis of the already known Neptune "non-Plus" MC-ICP-MS instruments, but the installation of the CDD detectors and the addition of one more energy filter (called RPQ, "Retarding Potential Quadrupole") have added more flexibility and widened the range of applications. Note that the detector SEM IC1 can be used in two ways, either as SEM IC1C when the ion beam passes between the cups $\mathrm{L} 1$ and $\mathrm{H} 1$ before going through RPQ1 into the SEM IC1, or as SEM IC1B when the ion beam passes between the cups L4 and L5 on its way through RPQ1 into SEM IC1. This flexibility allows using SEM IC1 which is equipped with RPQ1 in combination with several other ion

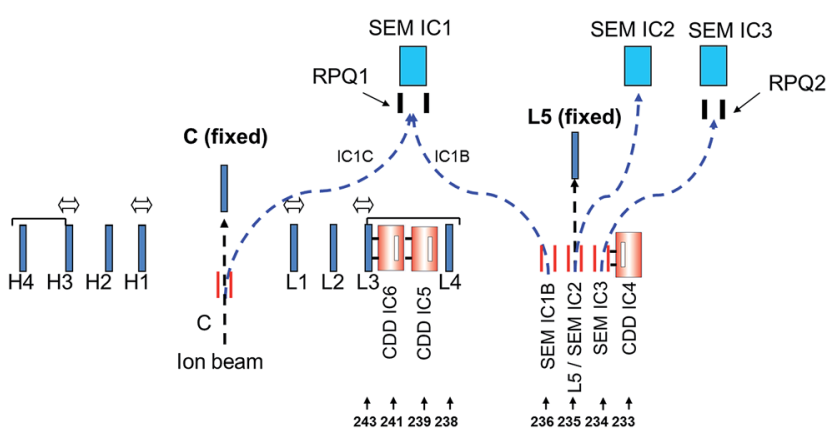

Fig. 2 Detector configuration of the Neptune Plus ${ }^{T M}-2$ MC-ICP-MS. counters, such as SEM IC2, SEM IC3 (equipped with RPQ2), CDD IC4 and even CDD IC5.

The "L5 ion counter package" includes a series of four ion counters with one mass unit spacing in the U-Pu mass region, but without the need for using CDEM detectors. This configuration allows to measure low level uranium samples by simultaneous ion counting, the minor isotopes ${ }^{234} \mathrm{U}$ and ${ }^{236} \mathrm{U}$ being detected in ion counters SEM IC3 and SEM IC1 which are equipped with energy filters RPQ2 and RPQ1 for improved abundance sensitivity, while the major isotopes ${ }^{235} \mathrm{U}$ and ${ }^{238} \mathrm{U}$ are being detected in SEM IC2 and CDD IC5, respectively. CDD IC5 can be moved accordingly by moving cup L4 close to the ion beam slit for SEM 1C1B. In case one or both of the major ion beams are too intense for ion counters, the major isotopes can alternatively be measured using Faraday cups, e.g. ${ }^{235} \mathrm{U}$ in cup L5 and ${ }^{238} \mathrm{U}$ in cup L4, which can be moved accordingly. Also ${ }^{233} \mathrm{U}$ from a ${ }^{233} \mathrm{U}$ spike material for IDMS can be detected simultaneously in ion counting mode using CDD IC4.

For low level $\mathrm{Pu}$ measurements, also simultaneous ion counting is possible, although not for all isotopes from ${ }^{238} \mathrm{Pu}$, ${ }^{239} \mathrm{Pu},{ }^{240} \mathrm{Pu},{ }^{241} \mathrm{Pu},{ }^{242} \mathrm{Pu}$ to ${ }^{244} \mathrm{Pu}$ in one step. A two-step "multidynamic" mass cycle would be on option. More information about the type of samples to be measured for nuclear safeguards purposes, their typical concentrations and isotopic compositions, can be found in. ${ }^{7}$

\section{The static measurement procedure}

For the linearity testing using the reference materials from the IRMM-072 series the ${ }^{233} \mathrm{U}$ count rate was measured at various intensity levels on the ion counting detector to be investigated, whereas the ${ }^{235} \mathrm{U}$ and ${ }^{238} \mathrm{U}$ isotopes were detected using Faraday cups at a uniform intensity level for each of the measured reference materials of the series. The inter-calibration of the ion counting detector versus the Faraday cup system is in each case performed internally by the additional detection of ${ }^{235} \mathrm{U}$ with the ion counter of interest and one Faraday cup. Note that all Faraday cups were inter-calibrated using the so-called electronic gain calibration procedure.

The principle of the static procedure is explained in the following for the CDD IC5 detector of the Neptune-1. The entire static procedure consisted of a series of measurements of the IRMM-072/1-7 reference materials (see Table 2). The remaining materials IRMM-072/8-15 have smaller ${ }^{233} \mathrm{U} /{ }^{238} \mathrm{U}$ ratios and too small ${ }^{233} \mathrm{U}$ count rates, which are not useful for this purpose. The isotope ratio measurement methods usually consisted of 5 blocks of 10 mass cycles. By measuring the reference materials from IRMM-072/1 to IRMM-072/7, the ${ }^{233} \mathrm{U}$ intensity is covering the entire range of count rates from $c a .800000 \mathrm{cps}$ down to a about $10000 \mathrm{cps}$. The ${ }^{235} \mathrm{U}$ and ${ }^{238} \mathrm{U}$ intensities on the Faraday cups were kept a uniform level of about $13 \mathrm{mV}$, corresponding to about $800000 \mathrm{cps}$ on CDD IC5.

As indicated on the certificate, the total $U$ concentrations for each material of the IRMM-072/1-15 series are $1 \mathrm{mg} U$ per $\mathrm{mL}$ solution $( \pm 1 \%)$, which is too high for a direct measurement on MC-ICP-MS instruments, therefore all solutions were diluted to a concentration of about $0.6 \mathrm{ng} U$ per $\mathrm{g}$. A standard sample 
Table 1 Tuning parameters of the Neptune-1 and Neptune-2 MC-ICP-MS instruments. If not otherwise indicated, values refer to both N-1 and $\mathrm{N}-2$ measurements

Instrumental parameters

\section{Sample introduction}

Standard sample introduction system

Nebulizer

Sample uptake rate

Sample matrix

Sample gas

Sample uptake time

Wash time

MC-ICP-MS

RF power

Auxiliary gas

Cool gas

Cones

$\mathrm{m} / \mathrm{z}$ monitored

Mass separation

Resolution $m / \Delta m$

Detection systems

Voltages applied to RPQ 1 (SEM IC1)

Decelerator voltage

Suppressor voltage

Acquisition parameters

Number of locks

Cycles/block

\author{
Cyclone/double pass spray chamber \\ PFA 100 (self-aspirating) \\ $100 \mu \mathrm{L} \mathrm{m^{-1 }}$ \\ $2 \%(\mathrm{~m} / \mathrm{m}) \mathrm{HNO}_{3}$ \\ $1 \mathrm{~L} \mathrm{~min}^{-1}(\mathrm{~N}-1), 0.9 \mathrm{~L} \mathrm{~min}^{-1}(\mathrm{~N}-2)$ \\ $180 \mathrm{~s}$ \\ $300 \mathrm{~s}$
}

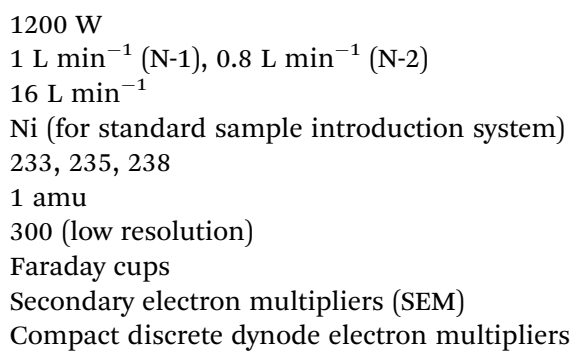
(CDD)

$8250.0 \mathrm{~V}$ (only used on $\mathrm{N}-1$ for this work) $9963.2 \mathrm{~V}$ (only used on $\mathrm{N}-1$ for this work)

5

10 introduction system with a cyclone/double pass spray chamber was used with a PFA 100 (uptake rate: $100 \mu \mathrm{L} \mathrm{min}^{-1}$ ) nebulizer. The wash solution was $4 \%(\mathrm{v} / \mathrm{v}) \mathrm{HNO}_{3}$, the wash time was 300 seconds, the sample uptake time was 180 seconds. The tuning parameters of the Neptune-1 and Neptune-2 MC-ICP-MS instruments are given in Table 1.

As shown in Table 2, due to the fact that the $\mathrm{U}$ concentrations are the same, the ${ }^{235} \mathrm{U}$ concentrations differ within the series. This means that prior to the linearity test measurement the total uranium concentrations of the diluted sample solutions for IRMM-072/1-7 had to be adjusted in a way, that the ${ }^{235} \mathrm{U}$ (and ${ }^{238} \mathrm{U}$ ) amount concentrations were similar for each of them and the ${ }^{235} \mathrm{U}$ and ${ }^{238} \mathrm{U}$ intensities could be kept at the required uniform level. Looking at IRMM-072/1 towards IRMM-072/7, the ${ }^{235} \mathrm{U} /{ }^{238} \mathrm{U}$ ratio is about constant but the ${ }^{233} \mathrm{U} /{ }^{238} \mathrm{U}$ ratio is decreasing. Therefore the relative abundance of ${ }^{235} \mathrm{U} / \mathrm{U}$ is increasing, and as a consequence the samples needed to be increasingly diluted in comparison to IRMM-072/1. This would apply in a similar manner if the IRMM-073 and IRMM-074 series were used.

For each dead time measurement sequence, the solutions of IRMM-072/1-7 were measured in an automated manner in a 'forth and back' fashion, i.e. starting with IRMM-072/7, continuing towards IRMM-072/1, measuring IRMM-072/1 again and going back towards IRMM-072/7. This is the reason why for each count rate of ${ }^{233} \mathrm{U}$ two data points are shown in the

Table 2 Certified isotope ratios for IRMM-072 (uncertainties with coverage factor $k=2$ ), relative abundances ${ }^{235} \mathrm{U} / \mathrm{U}$, and required dilution factors

\begin{tabular}{|c|c|c|c|c|c|}
\hline & $\begin{array}{l}{ }^{233} \mathrm{U} /{ }^{235} \mathrm{U} \\
( \pm 0.03 \%)\end{array}$ & $\begin{array}{l}{ }^{233} \mathrm{U} /{ }^{238} \mathrm{U} \\
( \pm 0.03 \%)\end{array}$ & $\begin{array}{l}{ }^{235} \mathrm{U} /{ }^{238} \mathrm{U} \\
( \pm 0.02 \%)\end{array}$ & ${ }^{235} \mathrm{U} / \mathrm{U}$ & $\begin{array}{l}\text { Required dilution } \\
\text { relative to IRMM-072/1 }\end{array}$ \\
\hline IRMM-072/1 & 1.00033 & 0.99136 & 0.99103 & $33.2 \%$ & 1.00 \\
\hline IRMM-072/2 & 0.69967 & 0.69385 & 0.99168 & $36.9 \%$ & 1.11 \\
\hline IRMM-072/3 & 0.49985 & 0.49591 & 0.99212 & $39.9 \%$ & 1.20 \\
\hline IRMM-072/4 & 0.29987 & 0.29763 & 0.99256 & $43.3 \%$ & 1.30 \\
\hline IRMM-072/5 & 0.100014 & 0.099313 & 0.99299 & $47.5 \%$ & 1.43 \\
\hline IRMM-072/6 & 0.050091 & 0.049746 & 0.99310 & $48.6 \%$ & 1.46 \\
\hline IRMM-072/7 & 0.019994 & 0.019857 & 0.99317 & $49.3 \%$ & 1.48 \\
\hline
\end{tabular}


Table 3 Mass cycle arrangement for the static procedure for CDD IC5, on Neptune-1

\begin{tabular}{lllll}
\hline Step & CDD IC5 & Cup L3 & Cup L2 & Cup L1 \\
\hline 1 & ${ }^{233} \mathrm{U} / 10000-800000 \mathrm{cps}$ & ${ }^{235} \mathrm{U} / 13 \mathrm{mV}$ & & ${ }^{238} \mathrm{U} / 13 \mathrm{mV}$ \\
2 & ${ }^{235} \mathrm{U} / 800000 \mathrm{cps}$ & & ${ }^{238} \mathrm{U} / 13 \mathrm{mV}$ &
\end{tabular}

following figures within the "Results" section. A blank measurement was performed before each sample by measuring the same $2 \% \mathrm{HNO}_{3}$ solution which was used for diluting the IRMM-072 material. Blank correction was performed by using the Neptune Plus ${ }^{\mathrm{TM}}$ software.

The mass cycle arrangement for the CDD IC5 detector is shown in Table 3. The advantage of this mass cycle arrangement is the fact that the efficiency (yield) of the CDD detector versus the Faraday cup system can be calculated as a ratio of ratios, rather than a ratio of intensities. Thereby only statically measured ratios are used and no dynamic (peak-jumping) ratios, which makes this procedure independent on plasma instabilities and therefore advantageous for ICP-MS.

The ${ }^{233} \mathrm{U} /{ }^{235} \mathrm{U}$ ratio is first corrected for the ion counter efficiency as shown in eqn (1):

$$
\left(\frac{{ }^{233} \mathrm{U}}{{ }^{235} \mathrm{U}}\right)_{\mathrm{CAL}}=\left(\frac{{ }^{233} \mathrm{U}}{{ }^{235} \mathrm{U}}\right)_{\mathrm{STEP} 1}\left[\left(\frac{{ }^{235} \mathrm{U}}{{ }^{238} \mathrm{U}}\right)_{\mathrm{STEP} 1} /\left(\frac{{ }^{235} \mathrm{U}}{{ }^{238} \mathrm{U}}\right)_{\text {STEP } 2}\right]
$$

The mass fractionation of the ${ }^{233} \mathrm{U} /{ }^{235} \mathrm{U}$ ratio is corrected internally using eqn (2), using the ${ }^{235} \mathrm{U} /{ }^{238} \mathrm{U}$ ratio measured by Faradays cups in Step 1:

$\left(\frac{{ }^{233} \mathrm{U}}{{ }^{235} \mathrm{U}}\right)_{\mathrm{CORR}}=\left(\frac{{ }^{233} \mathrm{U}}{{ }^{235} \mathrm{U}}\right)_{\mathrm{CAL}}\left[\left(\frac{{ }^{235} \mathrm{U}}{{ }^{238} \mathrm{U}}\right)_{\mathrm{CERTIFIED}} /\left(\frac{{ }^{235} \mathrm{U}}{{ }^{238} \mathrm{U}}\right)_{\mathrm{STEP} 1}\right]^{2 / 3}$

\section{Results and discussion}

The result for the dead time of the entire CDD IC5 detector and counting system can be calculated as the slope of a regression line, as shown in Fig. 3, for which the quantity $\left\{\left({ }^{233} \mathrm{U} /{ }^{235} \mathrm{U}\right)_{\mathrm{CORR}} /\right.$ $\left.\left({ }^{233} \mathrm{U} /{ }^{235} \mathrm{U}\right)_{\mathrm{CERT}^{-1}}\right\}$ (i.e. relative difference to certified ratio) is plotted versus the count rate of ${ }^{233} \mathrm{U}$. Note that the measured ratios are corrected for mass fractionations as explained, but should not be corrected for the dead time effect by the instrument software, or the dead time value has to be set to zero. The measurement with the highest count rate of ${ }^{233} \mathrm{U}$ is the result for the IRMM-072/1, which is a $1 / 1 / 1$ mixture of ${ }^{233} \mathrm{U} /{ }^{235} \mathrm{U} /{ }^{238} \mathrm{U}$. The deviation of the value $\left({ }^{233} \mathrm{U} /{ }^{235} \mathrm{U}\right)_{\mathrm{CORR}}$ from the certified value $\left({ }^{233} \mathrm{U} /{ }^{235} \mathrm{U}\right)_{\mathrm{CERT}}$ should be zero within the uncertainties, because in this case the dead time effects for ${ }^{233} \mathrm{U}$ and ${ }^{235} \mathrm{U}$ are the same. The fact that this was actually observed in the measurement indicates that there is no mass related residual bias.

By measuring a sequence through the IRMM-072 series, the ${ }^{233} \mathrm{U}$ count rate is changing from one material to the next, which causes the dead time effect for the detection of ${ }^{233} \mathrm{U}$ to differ from the (maximum) dead time effect experienced for the ${ }^{235} \mathrm{U}$ detected in CDD IC5 in Step 2 of the mass cycle. The measured data for CDD IC5 in Fig. 3 are well fitted by the regression line. The calculated result for the dead time is $\tau=(66.3 \pm 1.9) \mathrm{ns}$ (coverage factor $k=2$ ), which is close the electronically determined dead time of about $70 \mathrm{~ns}$. This confirms that there is no evidence for an inherent SEM non-linearity, and the 'true' dead time can be derived from the regression.

The uncertainty of the dead time is calculated according to the $\mathrm{GUM}^{8}$ with a coverage factor of $k=2$ and take into account the uncertainties of all individual measurements within the series. Since some of those, in particular for low count rates close to the instrumental background, don't overlap with the regression line, the uncertainties for those measurements were augmented accordingly and the uncertainty of the slope re-calculated. Based on the uncertainty of this dead-time of

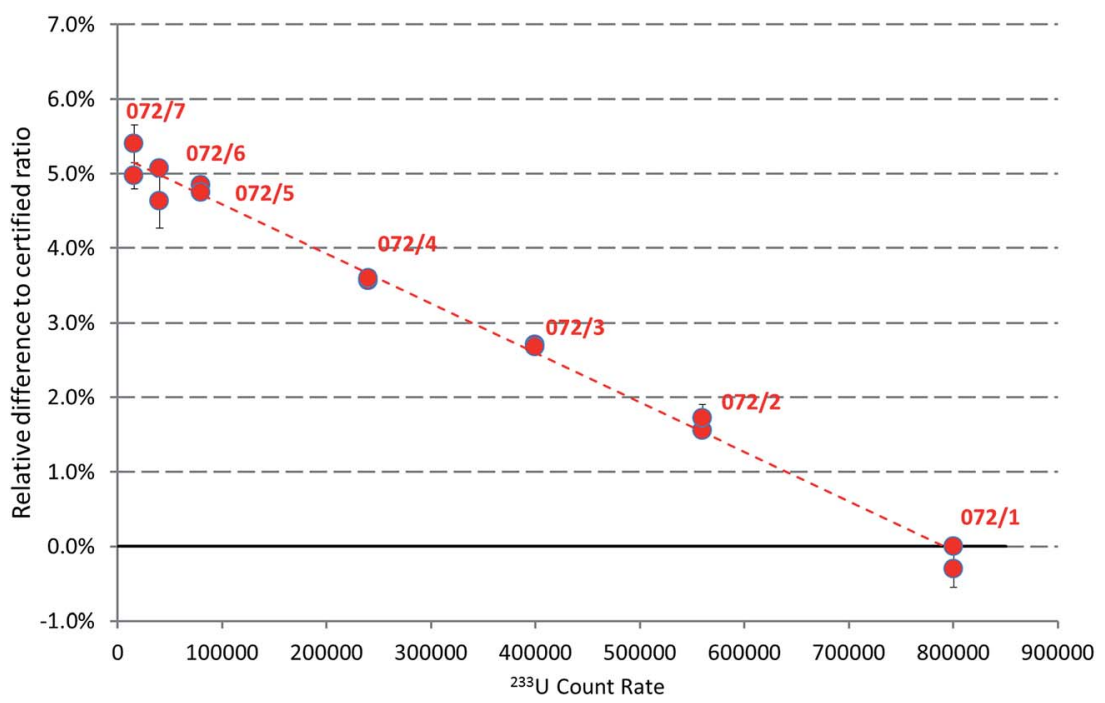

Fig. 3 Linearity test of CDD detector IC5 using the IRMM-072 series. The dead time calculated from the slope of the regression line is $\tau=(66.3 \pm$ 1.9) ns $(k=2)$. 
about $2 \mathrm{~ns}$, for isotope ratio measurements with count rates at the level of $500000 \mathrm{cps}$, the uncertainty arising from the dead time correction would be below $0.1 \%$.

The pulse amplifiers used on all Neptune ${ }^{\mathrm{TM}}$ MC-ICP-MS have three settings available for the dead time, their nominal values are $20 \mathrm{~ns}, 50 \mathrm{~ns}$ and $70 \mathrm{~ns}$, which are close to the electronically defined and roughly confirmed values. For the CDD detectors of the Neptune ${ }^{\mathrm{TM}}$, typically the longest available dead time is chosen in the factory in order to circumvent small pulses arising from reflections occurring within the rather long BNC cables between the detectors themselves and the pulse amplifiers. It has to be noted, that the measurement result of the dead time for this CDD IC5 detector is significantly different from the nominal value of $70 \mathrm{~ns}$. Using the nominal dead time which is about 4 ns higher than the SI traceable

Table 4 Mass cycle arrangement for the static procedure for SEM IC1C, on Neptune-1

\begin{tabular}{lllll}
\hline Step & SEM IC1C $^{a}$ & Cup H1 & Cup H2 & Cup H3 \\
\hline 1 & ${ }^{233} \mathrm{U} / 10000-800$ 000 cps & ${ }^{235} \mathrm{U} / 13 \mathrm{mV}$ & & ${ }^{238} \mathrm{U} / 13 \mathrm{mV}$ \\
2 & ${ }^{235} \mathrm{U} / 800000 \mathrm{cps}$ & & ${ }^{238} \mathrm{U} / 13 \mathrm{mV}$ &
\end{tabular}

" The detector notation "IC1C" expresses that the ion beam passes between L1 and H1 before going into SEM IC1.

Table 5 Mass cycle arrangement for the static procedure for SEM IC1B, on Neptune-1

\begin{tabular}{llllll}
\hline Step & SEM IC1B $^{a}$ & Cup L4 & CDD IC5 & Cup L3 & Cup L2 \\
\hline 1 & ${ }^{233} \mathrm{U}$ & ${ }^{235} \mathrm{U}$ & & ${ }^{238} \mathrm{U}$ & \\
2 & ${ }^{235} \mathrm{U}$ & & ${ }^{238} \mathrm{U}$ & & \\
3 & & & ${ }^{235} \mathrm{U}$ & & ${ }^{238} \mathrm{U}$
\end{tabular}

${ }^{a}$ The detector notation "IC1B" expresses that the ion beam passes between L4 and L5 before being deflected into SEM IC1. measured value would lead to a difference of $0.2 \%$ for count rates of $500000 \mathrm{cps}$. This can cause significant biases in isotope ratio measurements.

\section{The static procedures for SEM IC1C and SEM IC1B on Neptune-1}

For one of the other electron multipliers of the Neptune Plus ${ }^{\mathrm{TM}}-1$ MC-ICP-MS, for SEM IC1 - a classical normal size discrete dynode secondary electron multiplier - the mass cycle arrangement is similar to CDD IC5, as shown in Table 4. In case SEM IC1 is used as SEM IC1C, the ion beam passes between L1 and $\mathrm{H} 1$ to reach SEM IC1, while in case SEM IC1 is used as SEM IC1B the ion beam passes between L4 and L5 Faraday cups. In both cases, the same SEM detector is used (see Fig. 1), therefore the dead time results are expected to agree.

If SEM IC1B is used instead of SEM IC1C, the ion beam passes between the Faraday cups L4 and L5, therefore the mass cycle arrangement has to be very much different as shown in Table 5 . The count rates are the same as already shown in the previous Tables 3 and 4. The reason for adding Step 3 in Table 5 is the need for cross-calibration of the ion counter CDD IC5 with the Faraday cup system. This is achieved by static measurements of the ${ }^{235} \mathrm{U} /{ }^{238} \mathrm{U}$ ratio in Step 3 in comparison with the ${ }^{235} \mathrm{U} /{ }^{238} \mathrm{U}$ isotope ratio measured in Step 1 on Faraday cups only.

In this mass cycle arrangement the ion counter CDD IC5 is used for detecting ${ }^{235} \mathrm{U}$ and ${ }^{238} \mathrm{U}$ without correction for the dead time effect, but this has no influence because the ${ }^{235} U /{ }^{238} U$ isotope ratio is close to one and therefore the count rates are similar. Fig. 4 shows a linearity test of SEM IC1B, according to the mass cycle arrangement in Table 5. Note that for this measurement cup L4 has to be moved two mass units away from the beam position of IC1B.

Due to several small deviations for individual measurements, the uncertainties had to be slightly augmented, in this case in a uniform way, to achieve an overlap with the regression line as well as an overlap of the result for IRMM-072/1 (with $\left.{ }^{233} U /{ }^{235} U /{ }^{238} U=1 / 1 / 1\right)$ with the zero difference line. This is

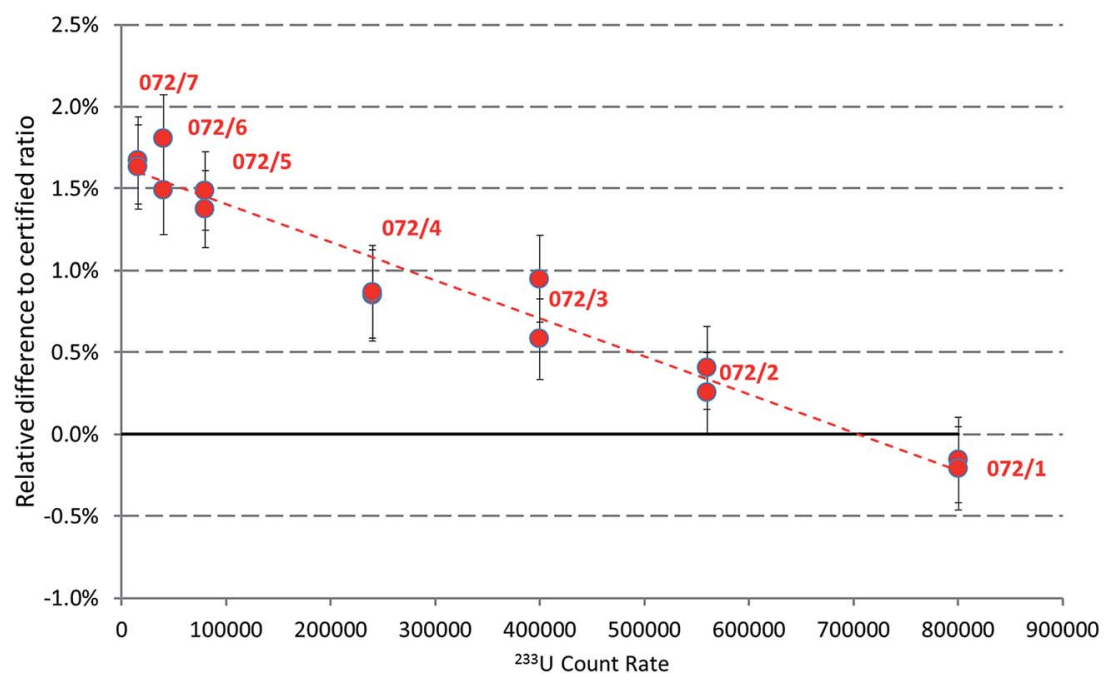

Fig. 4 Linearity Test of SEM IC1B using the IRMM-072 series. 
considered an important additional requirement for a correctly performed dead time measurement sequence, because the measurement of the sample IRMM-072/1 (with all count rates at ca. $800000 \mathrm{cps}$ ) should be independent on any dead time effect and should be in agreement with the certified value. In case of CDD IC5 and also for SEM IC1C, this requirement was already fulfilled without augmenting the uncertainties.

For the determination of the dead time of SEM IC1, several measurements were performed as SEM IC1C and SEM IC1B, as shown in Fig. 5. Some of the measurements were performed with the energy filter RPQ1 in operation, and some without, but this seemed to have no significant influence on the dead time result. Also the blank correction was found to have no significant influence.

The combined result for the dead time of SEM IC1 is $\tau=$ $(23.57 \pm 0.87) \mathrm{ns}(k=2)$. Note that the measured dead time is much lower compared to CDD IC5. This is due to the different dead time setting at the pulse amplifier, which is justified due to the quite short cable connection between the detector itself and the pulse amplifier. But in this case again the measured dead time value differs from the nominal value of $20 \mathrm{~ns}$. For this combined result for SEM IC1 only the measurements for SEM IC1C, applying the easier mass cycle arrangement in Table 4, were taken into account. The measurements for SEM IC1B applying the mass cycle arrangement in Table 5 required one more measurement step and were also compromised by the need for augmenting the individual uncertainties, which caused the uncertainties of the dead time results to increase. All results for SEM IC1C and SEM IC1B agree well with each other, because in each case the same detector, namely SEM IC1, is investigated, but however, in different ways with different performances. The results for SEM IC1B are rather considered as confirmation for the combined result calculated from those obtained for SEM IC1C.

\section{The static procedure for SEM IC2 on Neptune-1}

The mass cycle arrangement for the linearity testing of SEM IC2 is shown in Table 6. Note that according to the detector arrangement in Fig. 1, the ion beam can be switched between

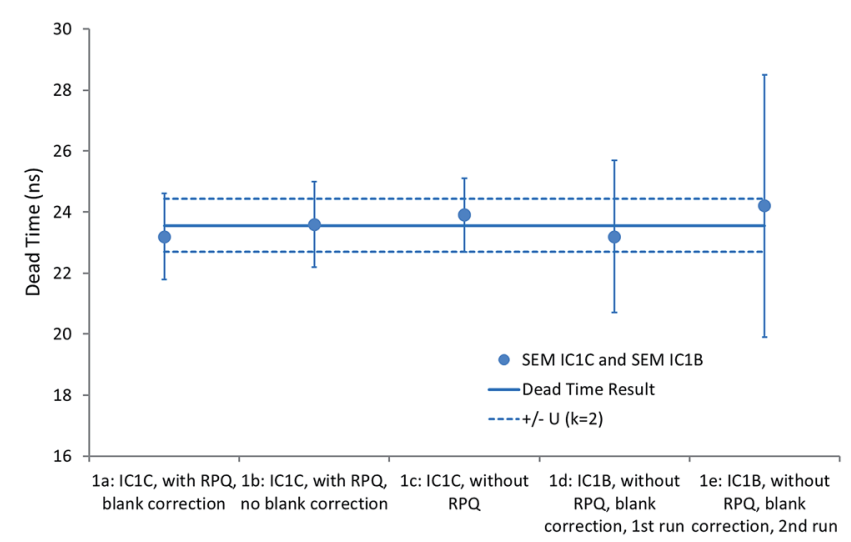

Fig. 5 Summary of linearity tests for SEM IC1, used as SEM IC 1 C and SEM IC1B.
Table 6 Mass cycle arrangement for the static procedure for SEM IC2, on Neptune-1

\begin{tabular}{lllll}
\hline Step & IC2/Cup L5 ${ }^{a}$ & Cup L4 & CDD IC5 & Cup L3 \\
\hline 1 & ${ }^{233} \mathrm{U}$ on IC2 & ${ }^{235} \mathrm{U}$ & & ${ }^{238} \mathrm{U}$ \\
2 & ${ }^{235} \mathrm{U}$ on IC2 & & ${ }^{238} \mathrm{U}$ & \\
3 & ${ }^{235} \mathrm{U}$ on Cup L5 & & ${ }^{238} \mathrm{U}$ &
\end{tabular}

${ }^{a}$ The ion beam is switched from IC2 into cup L5 in Step 3, in order to calibrate CDD IC5 against the Faraday cup system.

Faraday cup L5 and SEM IC2 using a deflection voltage. For the procedure for SEM IC2 this is very advantageous, because it allows the ion counter CDD IC5 used in Step 2, to be cross-calibrated against cup L5 of the Faraday cup system in Step 3. For SEM IC2, one linearity measurement sequence was performed, the result for the dead time was $\tau=(21.4 \pm 2.9) \mathrm{ns}$ $(k=2)$. Note that for this measurement cup L4 was positioned only two mass units away from the position SEM IC2, which means cup L4 had to be moved close to the ion beam slit for IC1B.

\section{The static procedure for SEM IC3 on Neptune-1}

The procedure for SEM IC3, is the most complicated one on Neptune-1. In order to cross-calibrate all used ion counters against the Faraday cup system, one more step is necessary in the mass cycle arrangement (see Table 7). SEM IC3 is calibrated against the Faraday cup system in Step 3 using the ${ }^{235} U /{ }^{238} U$ ratio, which makes it necessary to move cup 4 close to the IC1B ion beam slit. Furthermore, the detector SEM IC1, used as SEM IC1B here, is needed for detecting ${ }^{235} \mathrm{U}$ in Step 1. SEM IC1B can only be calibrated using the isotope beam of ${ }^{233} \mathrm{U}$ in this procedure, because if ${ }^{235} \mathrm{U}$ was used in SEM IC1B, the ${ }^{238} \mathrm{U}$ beam would go between CDD IC5 and SEM IC3 and become unusable. In order to perform the calibration of SEM IC1B properly, the already determined dead time of SEM IC1 has to be applied to take into account the different count rates of ${ }^{235} \mathrm{U}$ in Step 1 and ${ }^{233} \mathrm{U}$ in Step 4 . The dead-time corrected ${ }^{233} \mathrm{U} /{ }^{235} \mathrm{U}$ ratio in Step 4 is then compared to the certified ${ }^{233} \mathrm{U} /{ }^{235} \mathrm{U}$ ratio in order to calculate the efficiency correction factor (yield) for SEM IC1B. For SEM IC3 two linearity measurement sequences were performed, the combined result for the dead time is $\tau=(16.5 \pm 2.0)$ ns $(k=2)$.

Table 7 Mass cycle arrangement for the static procedure for SEM IC3 on Neptune-1

\begin{tabular}{lllllll}
\hline Step & SEM IC3 & SEM IC1B $^{a}$ & Cup L4 & CDD IC5 & Cup L3 & Cup L2 \\
\hline 1 & ${ }^{233} \mathrm{U}$ & ${ }^{235} \mathrm{U}$ & & & & \\
2 & & & ${ }^{235} \mathrm{U}$ & & ${ }^{238} \mathrm{U}$ & \\
3 & ${ }^{235} \mathrm{U}$ & & ${ }^{238} \mathrm{U}$ & & & \\
4 & & ${ }^{233} \mathrm{U}$ & & ${ }^{235} \mathrm{U}$ & & ${ }^{238} \mathrm{U}$
\end{tabular}

${ }^{a}$ The detector notation "IC1B" expresses that the ion beam passes between L4 and L5 before being deflected into SEM IC1. 
Table 8 Mass cycle arrangement for the static procedure for CDD IC4 on Neptune-1

\begin{tabular}{lllllll}
\hline Step & CDD IC4 & Cup L5 & SEM IC1B $^{a}$ & Cup L4 & CDD IC5 & Cup L2 \\
\hline 1 & ${ }^{233} \mathrm{U}$ & ${ }^{235} \mathrm{U}$ & & ${ }^{238} \mathrm{U}$ & & \\
2 & ${ }^{235} \mathrm{U}$ & & ${ }^{238} \mathrm{U}$ & & & \\
3 & & & ${ }^{235} \mathrm{U}$ & & ${ }^{238} \mathrm{U}$ & \\
4 & & & & & ${ }^{235} \mathrm{U}$ & ${ }^{238} \mathrm{U}$
\end{tabular}

${ }^{a}$ The detector notation "IC1B" expresses that the ion beam passes between L4 and L5 before being deflected into SEM IC1.

\section{The static procedure for CDD4 IC4, on Neptune-1}

The mass cycle arrangement for CDD IC4 is shown in Table 8 below. The reason for performing Steps 3 and 4 are given by the need for cross-calibrations of the ion counters SEM IC1B and CDD IC5, performed by static measurements of the ${ }^{235} \mathrm{U} /{ }^{238} \mathrm{U}$ ratio, through comparison with the ${ }^{235} \mathrm{U} /{ }^{238} \mathrm{U}$ isotope ratio measured in Step 1 on Faraday cups only.

The dead time result for CDD IC4 using this mass cycle arrangement is $\tau=(67.6 \pm 2.8) \mathrm{ns}(k=2)$, which has a two times larger uncertainty than SEM IC1C or CDD IC5, and also takes twice as much measurement time. As an alternative, the following approach was taken. Starting with the assumption that the dead time of any type of secondary electron multiplier detector - standard size SEM or CDD - itself is much shorter, possibly at the level of $<10 \mathrm{~ns}$, compared to the (uniformed and enlarged) dead time of the pulse amplifier, the dead time of the entire system is given by the dead time of the pulse amplifier only. As a consequence, the true dead time value (not the nominal one) of the pulse amplifier currently used for CDD IC4 could also be determined using measurements on the detector CDD IC5. For doing this, at the detector flange output sockets for CDD IC4 and CDD IC5, the cables going to the respective pulse amplifiers would have to be exchanged with each other. Thereby the incoming ions are detected and amplified in CDD IC5, but the output pulses are counted by the pulse counting system of CDD IC4. All dead time relevant components of CDD IC4 (cables, connectors, amplifier, discriminator) and CDD IC5 (negligible due to short detector dead time) are taken into account. This approach is called "amplifier cross-over" from here on.

After a successful dead time measurement, the cables can be exchanged back and thereby the pulse amplifiers re-connected as they were originally set-up. This approach would make the dead time measurement for the pulse amplifier of CDD IC4 as easy as it was performed already for CDD IC5 before (see Table 2).

The results of two replicate measurements performed in this way were $\tau=(66.7 \pm 1.9) \mathrm{ns}(k=2)$ and $\tau=(68.1 \pm 1.2) \mathrm{ns}$. These two results can be combined to an average of $\tau=(67.4 \pm 1.8) \mathrm{ns}$, which has a lower uncertainty but is in good agreement with the earlier result of $\tau=(67.6 \pm 2.8) \mathrm{ns}$, obtained using the more complex mass cycle shown in Table 8 and with the original connection of the CDD IC4 detector with its designated pulse amplifier. As a conclusion, it has been confirmed that the dead time of the combined system of a secondary electron multiplier with a pulse amplifier is only determined by the pulse amplifier. Therefore it is feasible to exchange pulse amplifiers between the secondary electron multipliers in a comfortable way ("amplifier cross-over"). For the dead time measurement of each pulse amplifier a secondary electron multiplier can be chosen which allows a straight forward measurement with an easy mass cycle arrangement.

For the new second Neptune Plus ${ }^{\mathrm{TM}}-2$ at the Nuclear Material Laboratory (NML) of the IAEA, which has three CDD detectors, the "amplifier cross-over" approach developed for Neptune Plus ${ }^{\mathrm{TM}}-1$ was used as well. The three CDD detectors were found to have dead time values significantly different from each other. As a conclusion, the electronic properties of the pulse amplifiers are different enough to result in significantly different dead time values for each amplifier. The nominal values from the manufacturer can only be considered as indicative.

\section{The static procedure for SEM1 IC1C on Neptune-2}

For SEM IC1C the dead time measurement was performed in a similar way as on Neptune-1 using the mass cycle arrangement in Table 4. The combined result for two measurement sequences was $\tau=(22.3 \pm 1.5) \mathrm{ns}(k=2)$. The measurement for SEM IC1B cannot be performed in a similar way as shown in Table 5, because on Neptune-2, the detector CDD IC6 is located between CDD IC5 and cup L3, so cup L3 cannot be used in a similar way. But it was already shown for Neptune-1, that the dead time for SEM IC1 is the same whether it is determined using SEM IC1C or SEM IC1B, and the result for SEM IC1C was chosen due to the easier mass cycle and smaller uncertainties of the results. Thus, for Neptune-2, the result obtained using SEM IC1C can be used as dead time for SEM IC1.

\section{The static procedure for CDD IC6 on Neptune-2}

Due to the different detector configuration, CDD IC6 on Neptune-2 can be investigated in a similar way as CDD IC5 on Neptune-1. This is shown in Table 9, the result of one measurement sequence was $\tau=(68.0 \pm 2.4) \mathrm{ns}(k=2)$.

\section{The static procedures for CDD IC4 and CDD IC5 on Neptune-2}

The dead time measurements for CDD IC4 and CDD IC5 on Neptune-2 were performed using the CDD detector IC6, each time connected to the pulse amplifiers and counting systems of CDD IC4 and CDD IC5, in a similar manner ("amplifier cross-over") as already done for CDD IC4 on Neptune-1. The results were $\tau=(65.6 \pm 2.4) \mathrm{ns}$ for CDD IC4 and $\tau=(65.9 \pm 3.0)$ ns (coverage factor $k=2)$ for CDD IC5.

Table 9 Mass cycle arrangement for the static procedure for CDD IC6 on Neptune-2

\begin{tabular}{lllll}
\hline Step & CDD IC6 & Cup L3 & Cup L2 & Cup L1 \\
\hline 1 & ${ }^{233} \mathrm{U}$ & ${ }^{235} \mathrm{U}$ & & ${ }^{238} \mathrm{U}$ \\
2 & ${ }^{235} \mathrm{U}$ & & ${ }^{238} \mathrm{U}$ &
\end{tabular}


The static procedures for SEM IC2, and SEM IC3, on Neptune-2

For the detectors SEM IC2 and SEM IC3 on Neptune-2 no suitable static measurement procedures could be applied. This is due to the presence of CDD IC6, which results in unavoidable mass distances larger than one mass unit between neighbouring detectors. Alternatively, some procedures using peakjumping measurements to achieve counter cross-calibrations could be considered, but this would cause much larger uncertainties due to plasma instabilities. This was observed already for some initial test measurements for SEM IC1B, SEM IC2 and SEM IC3 on Neptune-1, at an earlier time (in 2012) when it was not yet possible to move cup L4 close enough to the ion beam slit for IC1B.

An alternative suggestion could be to perform cross-over amplifier exchanges between SEM IC1, SEM IC2 and SEM IC3 on Neptune-2, in a similar way as performed between CDD IC4, CDD IC5 and CDD IC6. But due to the shorter dead time values of SEM IC1, SEM IC2 and SEM IC3, which are resulting from much shorter cable connections between the detectors and the pulse amplifiers, this cross-over cannot be achieved by simply swapping cables or making longer alternative cable connections. Instead, if the pulse amplifiers of SEM IC2 and SEM IC3 were to be investigated, the amplifiers would have to be physically moved and installed in place of the pulse amplifier of SEM IC1, and then measured as SEM IC1C.

This suggested procedure could first be validated on Neptune-1, for which the dead times of SEM IC2 and SEM IC3 have already been determined directly. If this was successful, it could be done on Neptune- 2 as well, and would probably be the most reliable way to determine the dead times of SEM IC2 and SEM IC3 on Neptune-2, except for using procedures with peakjumping and higher uncertainties. Unfortunately, the suggested procedures for SEM IC2 and SEM IC3 on Neptune-2 could not be tested within the frame of this paper, as it required an alteration of the hardware and significant time expenses that were not feasible with respect to the tight schedule of the MC-ICP-MS operation in the nuclear material laboratory. For now, the dead times of SEM IC2 and SEM IC3 are set as the nominal values of $20 \mathrm{~ns}$, but as already mentioned the nominal values are not always very reliable.

\section{Summary and conclusions}

For the new two Neptune Plus ${ }^{\mathrm{TM}}$ MC-ICP-MS instruments at the IAEA, the linearity of all SEM and CDD ion counting detectors was investigated by measurement sequences of the IRMM-072 series, and the dead time values were determined. The results are shown in Table 10 and Fig. 6 below.

The following concluding statements can be made from the results:

(1) For all investigated detectors, a linear relationship between the dead-time-un-corrected isotope ratios and the ion beam intensity (or count rate) was confirmed. The slopes of the linear regressions were used to calculate new dead time values which were close (at least within $\pm 5 \mathrm{~ns}$ ) to the nominal values set in the factory. The measured new dead time values are traceable to the SI due to the use of the gravimetrically prepared IRMM-072 series of reference materials.

(2) For the calculation of the dead time values from the linear regression, the uncertainties of individual measurements had to be slightly augmented in order to achieve the required overlaps for the measurement for IRMM-072/1 $\left({ }^{233} \mathrm{U} /{ }^{235} \mathrm{U}=1 / 1\right)$ with the certified value and for the individual results with the regression line. The latter represents an additional uncertainty component arising from the isotope ratio measurements, which compensates for the lack of a known uncertainty to be calculated from the external repeatability of individual measurements. But the individual measurements are only performed twice for each count rate intensity, which is not often enough to derive a reliable uncertainty component. Thus, the regression calculation is performed on all individual (rather than the average) measurement results, and the uncertainties augmented accordingly. The expanded uncertainties of the dead time results are at the level of 2-3 ns (coverage factor $k=2$ ). The uncertainty contributions from the used reference material play a minor role in the uncertainty budget, the dominant uncertainty contributions are arising from the counting statistics and amplifier noise within the individual measurements.

(3) Considering the uncertainties derived for the measurements, most of the dead time results disagree with their

Table 10 Results for dead time measurements for all SEM and CDD ion counting detectors of Neptune-1 and Neptune-2 at the IAEA laboratories

Instrument/detector

Dead time, measured using IRMM-072

Dead time, nominal value

\begin{tabular}{l} 
Instrument/detector \\
\hline Neptune-1, SEM IC1 \\
(SEM IC1C and SEM IC1B) \\
Neptune-1, SEM IC2 \\
Neptune-1, SEM IC3 \\
Neptune-2, SEM IC1C \\
Neptune-2, SEM IC2 \\
Neptune-2, SEM IC3 \\
Neptune-1, CDD IC4 \\
Neptune-1, CDD IC5 \\
Neptune-2, CDD IC4 \\
Neptune-2, CDD IC5 \\
Neptune-2, CDD IC6
\end{tabular}

Neptune-1, SEM IC1

(SEM IC1C and SEM IC1B)

Neptune-2, SEM IC1C

Neptune-2, SEM IC2

Neptune-1, CDD IC5

Neptune-2, CDD IC6

$$
\begin{aligned}
& \tau=(23.57 \pm 0.87) \mathrm{ns}(k=2) \\
& \tau=(21.4 \pm 2.9) \mathrm{ns}(k=2) \\
& \tau=(16.5 \pm 2.0) \mathrm{ns}(k=2) \\
& \tau=(22.3 \pm 1.5) \mathrm{ns}(k=2) \\
& \text { Not measured } \\
& \text { Not measured } \\
& \tau=(67.4 \pm 1.8) \mathrm{ns}(k=2) \\
& \tau=(66.3 \pm 1.9) \mathrm{ns}(k=2) \\
& \tau=(65.6 \pm 2.4) \mathrm{ns}(k=2) \\
& \tau=(65.9 \pm 3.0) \mathrm{ns}(k=2) \\
& \tau=(68.0 \pm 2.4) \mathrm{ns}(k=2)
\end{aligned}
$$




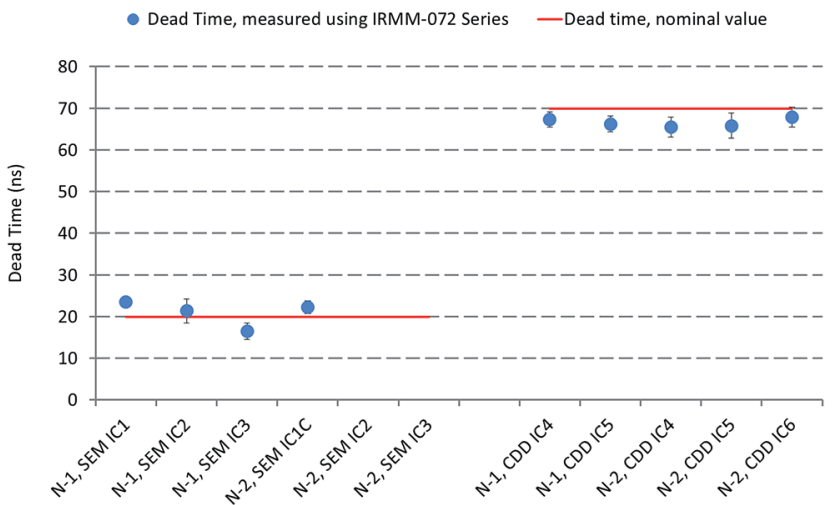

Fig. 6 Results for dead time measurements for all SEM and CDD ion counting detectors of Neptune-1 and Neptune-2 at the IAEA laboratories.

respective nominal values. This applies in particular for CDDs for which the nominal dead time setting of $70 \mathrm{~ns}$ was chosen because of possible signal reflections occurring within the long cables $(>1 \mathrm{~m}$ ) between the detectors and their pulse amplifiers. As a consequence, if only the nominal dead time values are used for dead time correction, this can cause significant biases in measured isotope ratios.

(4) It is important to note that the results of dead time measurements for the investigated ion counting detectors were found to depend only on the pulse amplifiers connected to them. Thus the dead time does not depend on the detector itself, unless it is larger than that of the pulse amplifier which is usually not the case and could be easily checked using an oscilloscope. The dead time does also not depend on the ion flight path towards the detector, which was concluded from the agreement between dead times for SEM IC1 being used as IC1C or IC1B. Furthermore the dead time does not depend on the presence or operation of an energy filter like an RPQ within the flight path. Due to the low blank contribution and long wash times, also the blank or possible memory effects did not significantly influence the dead time results.

(5) Since the dead time does not depend on the ion counting detector itself, it is not subject to performance changes or drifts as known for ion counting detectors themselves, such a loss of efficiency over time. This was confirmed by the agreement between earlier dead time measurements in 2012 with recent results in 2015 and 2016. Therefore a re-measurement of the dead time on a regular basis does not appear to be necessary.

(6) Due to the fact, that the dead times of the investigated detectors only depend on the pulse amplifiers connected to them, it was possible to exchange the pulse amplifiers against the detectors and thereby determine the (only pulse-amplifierdependent) dead times using the best choices of detectors from the measurement point of view. This "amplifier cross-over" principle was validated using the dead time measurements for CDD IC4 of Neptune-1 and led to a smaller uncertainty for the dead time result, and subsequently applied to CDD IC5 of Neptune-1, and CDD IC4, CDD IC5 and CDD IC6 of Neptune-2.
(7) For the detectors SEM IC2 and SEM IC3 of Neptune-2, it was not possible to apply measurement procedures without the use of dynamic (peak-jumping) ratios in order to cross-calibrate all needed detectors. It is suggested to apply the "amplifier cross-over" principle for those as well in the future, by directly installing the pulse amplifiers of SEM IC2 and SEM IC3 in place of that for SEM IC1 on Neptune-2, and perform a measurement of SEM IC1C. A validation of this approach could be done on Neptune-1, on which the dead times for the detectors SEM IC2 and SEM IC3 could already be determined in the direct way and without dynamic (peak-jumping) ratios for detector cross-calibration. The "amplifier cross-over" principle is also expected to lead to smaller uncertainties for the dead times of SEM IC2 and SEM IC3 on Neptune-1 due to the easier mass cycle arrangement when performing the measurements using SEM IC1C.

(8) It would certainly be more convenient for some sample measurements to have CDD detectors developed which allow one mass unit spacing on both the low and the high mass sides. But this could cause direct linearity tests to become even more complicated in some cases. However, due to the "pulse amplifier cross-over" principle this can be possibly resolved by choosing the most suitable ion counting detector for the dead time measurement of each pulse amplifier.

(9) The described measurement procedures can be performed not only using the IRMM-072 series (concentration $1 \mathrm{mg} \mathrm{U}$ per g) which is exhausted and not for sale any more, but for MC-ICP-MS measurements also the diluted version of it, the IRMM-073 series (concentration about $1.5 \mu \mathrm{g} \mathrm{U}$ per $\mathrm{mL}$ ), and the new IRMM-074 series (concentration about $100 \mathrm{ng} \mathrm{U}$ per $\mathrm{mL}$ ), can be used.

(10) For linearity testing and dead time determination of ion counters installed on TIMS instruments, the so-called "dynamic procedure" was recommended and described in detail in Richter $e t a l .{ }^{4}$ This is a peak-jumping procedure for one selected sample of the IRMM-072 (or -073, -074) series, e.g. IRMM-072(-073)/8 or IRMM-074/3 with a ${ }^{233} \mathrm{U} /{ }^{235} \mathrm{U}$ ratio of about $1 / 100$, to be performed at various count rate intensity levels, possibly within an automated sequence. The static procedure described within this paper was found to be also feasible for TIMS but more difficult to realize, because the requirement to measure at similar and time wise constant intensity levels for ${ }^{235} \mathrm{U}$ (and ${ }^{238} \mathrm{U}$ ) is more difficult to realize for samples on TIMS filaments, compared to sample solutions introduced into an ICP-MS instrument. On the other hand, the dynamic procedure recommended for TIMS, is not at all suitable for MC-ICP-MS, because the instability of the plasma would cause increased uncertainties.

Although not all ion counters of both Neptune ${ }^{\mathrm{TM}}$ MC-ICP-MS instruments at the IAEA have been investigated for their dead times yet, the procedures described in this paper and our conclusions can already be advantageous for users in other laboratories and even on other types and brands of MC-ICP-MS instruments. Other laboratories with a MC-ICP-MS instrument and a detector configuration similar to Neptune-1 are encouraged to perform the validation of the "amplifier cross-over" principle for SEM IC2 and SEMIC3. 


\section{Acknowledgements}

The authors would like to thank numerous colleagues, in particular A. Trinquier, C. Bouman, H. Bars and J. Schwieters from Thermo Fisher for helpful discussions and contributions. Furthermore we greatly appreciate the continued support from the EU support program to the IAEA.

\section{References}

1 K. J. R. Rosman, W. Lycke, R. Damen, R. Werz, F. Hendrickx, L. Traas and P. De Bievre, Int. J. Mass Spectrom. Ion Processes, 1987, 79, 61-71.

2 W. Lycke, P. De Bièvre, A. Verbruggen, F. Hendrickx and K. Rosman, Fresenius' Z. Anal. Chem., 1988, 331, 214-215.

3 A. Held, G. Van Baelen, F. Hendrickx, P. Taylor, A. Verbruggen and R. Wellum, Internal Report IRMM, GE/ R/IM/22/02, 2002.
4 S. Richter, A. Alonso, Y. Aregbe, R. Eykens, F. Kehoe, H. Kühn, N. Kivel, A. Verbruggen, R. Wellum and P. D. P. Taylor, Int. J. Mass Spectrom., 2009, 281, 115-125.

5 G. C. Eiden, A. M. Duffin, M. Liezers, J. D. Ward, J. W. Robinson, G. L. Hart, S. H. Pratt, K. W. Springer, A. J. Carman and D. C. Duckworth, International Conference on Advances in Nuclear Forensics: Countering the Evolving Threat of Nuclear and Other Radioactive Material Out of Regulatory Control, Vienna, 2014, IAEA-CN-218-134, http://www-pub.iaea.org/ MTCD/Publications/PDF/SupplementaryMaterials/P1706/ Technical_Session_3F.pdf, downloaded on 28 May 2016.

6 D. Tuttas, J. B. Schwieters, C. Bouman and M. Deerberg, Thermo Fisher Scientific Application Note No. 30192.

7 S. Boulyga, S. Konegger-Kappel, S. Richter and L. Sangély, J. Anal. At. Spectrom., 2015, 30, 1469-1489.

8 International Organisaton for Standardisation, Guide to the Expression of Uncertainty in Measurements, @ISO, Geneva, Switzerland, 1993, ISBN 92-67-10188-9. 